\title{
Scanning Tunnelling Spectroscopy of Periodic Nickel Nanoparticles
}

\author{
M. Kamiński ${ }^{a}$, B. Susea ${ }^{a, *}$, M. Giersig ${ }^{a, b}$ And M. Kandulski ${ }^{a}$ \\ ${ }^{a}$ Institute of Physics, Poznań University of Technology \\ Nieszawska 13A, 60-965 Poznań, Poland \\ b "CAESAR" Dept. Nanoparticle Technology \\ Ludwig-Erhard-Allee 2, 53175 Bonn, Germany
}

\begin{abstract}
Scanning tunneling microscopy/spectroscopy as well as atomic force microscopy were applied to study the non-structural and nanoelectronic properties of periodic nickel nanoparticles deposited on $n$-silicon substrates. Periodic nickel (Ni) nanoparticles were prepared by using nanosphere lithography and analyzed by scanning tunneling microscopy/spectroscopy and atomic force microscopy. By the evaporation of Ni perfectly ordered nanoparticles were produced and very good correlation between latex mask was observed. Finally, tunneling spectroscopy performed with non-magnetic tip yield information about local electronic properties of nanoscale structures at surface.
\end{abstract}

PACS numbers: 68.37.Ef, 61.48.+w

\section{Introduction}

It is well known that the size-dependent properties of metal nanoparticles depend on the atomic arrangement and their electronic structure. The scanning tunneling spectroscopy (STS) is complementary to topographic imaging by scanning tunneling microscope and it is a useful technique to obtain microscopic information on electronic states. The first spectroscopic investigation of surface states over specific atomic sites was made by Becker et al. [1], who used a lock-in technique to measure the differential conductivity of Si (111) $-7 \times 7$ surfaces. Stroscio, Feenstra and coworkers $[2,3]$ demonstrated the utility of presenting the current-voltage $(I-V)$ characteristics as $(\operatorname{d} \ln I / \mathrm{d} \ln V)$ versus $V$, showing that peaks in this quantity, for data obtained on the cleaved $\mathrm{Si}$ (111)-2 $\times 1$ surface, corresponded to the energy position at which peaks in the density of states (DOS) occur. In addition,

*corresponding author; e-mail: Susla@phys.put.poznan.pl 
many other systems have been studied using a technique similar to those introduced in these initial experiments. Unlike spectroscopes such as photoemission, the STS is capable of obtaining tunneling spectra of both the occupied and unoccupied electronic states.

We report the local electronic behavior of the periodic thin Ni nanoparticles deposited on variously oriented $n$-silicon substrates.

\section{Experimental details}

The Cryo SXM (OMICRON) scanning tunneling microscope has been used to study the topography and spectroscopic properties of the periodic thin $\mathrm{Ni}$ nanoparticles deposited on oriented (111) n-silicon substrates. The scanning tunneling microscopy/spectroscopy (STM/STS) experiments are performed at room temperature in ambient air conditions. Imaging was performed in a constant current mode $(1.0 \mathrm{nA})$ at a sample bias $+1.0 \mathrm{~V}$, using an electrolytic etching $\mathrm{W}$ tip. The scaling of the microscope was calibrated on a highly oriented pyrolytic graphite surface.

In spectroscopic mode $I-V$ characteristics are recorded simultaneously by the interrupted feedback loop. At every point, in the scanning tunneling microscope $X-Y$ scan, the feedback loop is turned of and within a limited time interval ( $210 \mu \mathrm{s}$ for both polarities), the $I-V$ curve is recorded for 80 discrete voltages in the range from -0.5 to $+0.5 \mathrm{~V}$. The scan takes about 18 minutes, because a large number of data is collected. The strength of the method is that the topographic image and spectroscopic data are correlated. The $I-V$ characteristics obtained in this way are stored (on a computer disc) and their derivative is obtained.

The large-scale, 2D arrays of perfectly ordered magnetic nanoparticles were prepared using nanosphere litography [4]. The preparation method is modified based on a self-assembly of latex particles on water surface. Using polystyrene particles, with a diameter from $100 \mathrm{~nm}$ to $1000 \mathrm{~nm}$, it was possible to prepare arrays over $1 \mathrm{~cm}^{2}$, large areas without any structural defects. By the evaporation of nickel, periodic magnetic particle arrays were produced. The process was performed in $2 \times 10^{-8}$ mbar vacuum. Evaporation rate was $0.8 \mathrm{~nm} / \mathrm{min}$. Nickel thin films in the range of 5-40 $\mathrm{nm}$ were evaporated. The thickness of the growing metal layer was estimated using oscillating quartz plate. After nickel evaporation process, some samples were covered by very thin $(5 \mathrm{~nm})$ gold film. The main advantages of this technique are: a short preparation time, a high level of hexagonal structure orientation and a possibility of application of large, monolayered masks directly onto any kind of surface.

\section{Result and discussion}

During the fabrication of periodic particles arrays, we used various techniques to investigate the structure. The preparation process was followed by opti- 
cal microscopy investigation, atomic force microscopy (AFM) or scanning electron microscopy (SEM) measurements [4]. A very good correlation between latex masks and evaporated Ni structure was observed.

Figure 1 shows a typical AFM image well-ordered.

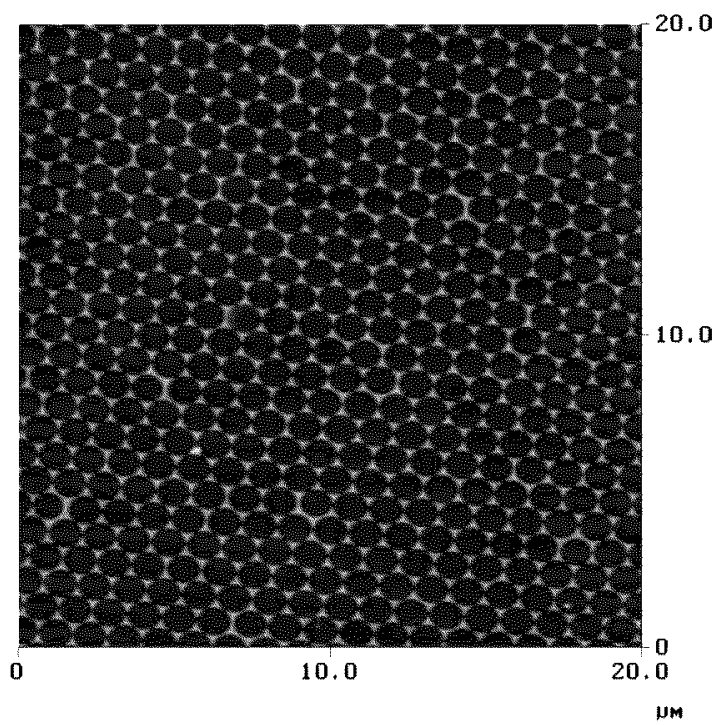

Fig. 1. AFM image of $0.015 \mu \mathrm{m}$ high Ni particles evaporated through $1.04 \mu \mathrm{m}$ polystyrene-latex beads mask shown in a very well-ordered structure.

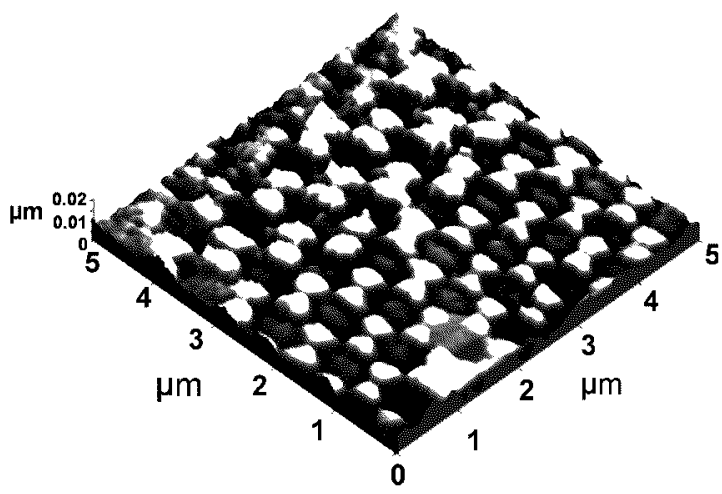

Fig. 2. Constant-current STM (3D) image of $0.015 \mu \mathrm{m}$ high nickel particles on the $n$-silicon substrate taken at $300 \mathrm{~K}$ - sample bias voltage $0.4 \mathrm{~V}$, tunneling current $I=1.2 \mathrm{nA}$.

Prepared quasi-triangular nanoclusters were also investigated using scanning tunneling microscope. The presented image (Fig. 2) shows well-ordered nickel particles exhibiting a homogeneous structure dictated by a hexagonal pattern of latex spheres. 


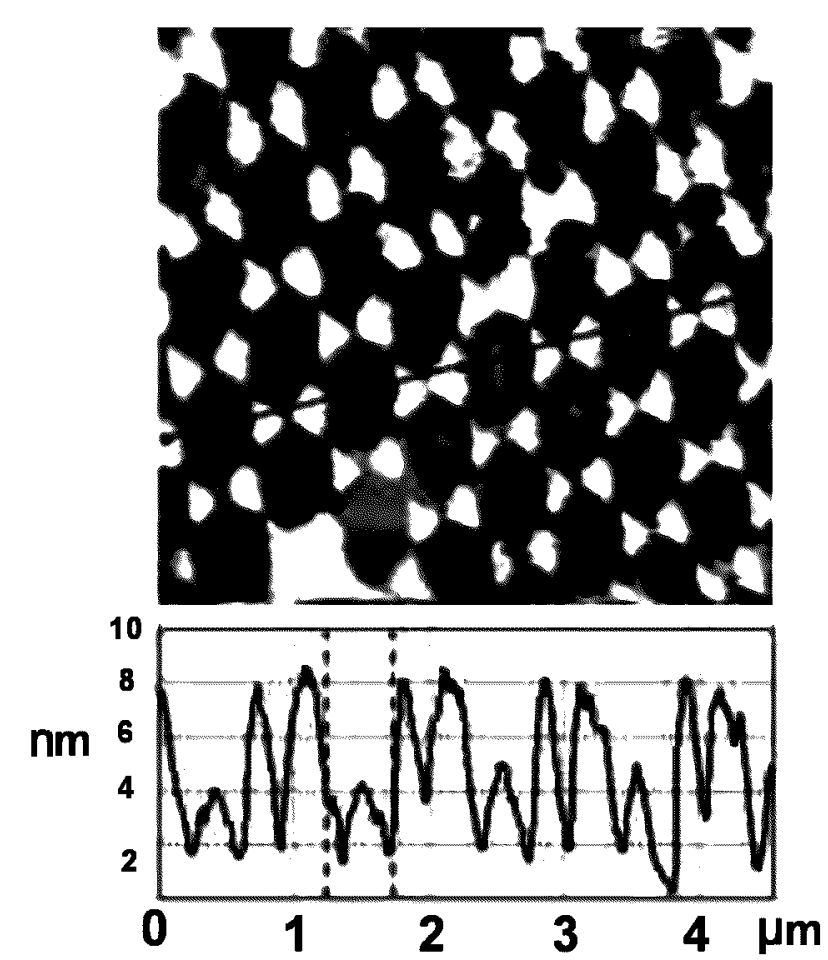

Fig. 3. The group of Ni clusters (white triangles to arrange in rosettes) deposited on Si substrate with profile of the surface corrugation measured along the line marked on the sample.

Figure 3 shows a topographic STM image of the quasi-two-dimensional (2D) nickel clusters. A line profile is shown in Fig. 3 and indicates the group of $\mathrm{Ni}$ clusters-white triangles of a diameter of $0.25 \mu \mathrm{m}$ and a height of $0.06 \mu \mathrm{m}$.

The idea of STS is to measure the dependence of the tunneling current $(I)$ on the applied voltage $(V)$. Then, the first derivative of the tunneling current with respect to voltage $(\mathrm{d} I / \mathrm{d} V)$ gives the local density of state (LDOS) of the sample [5-7]. The electronic structure of the tip is unknown, but it is constant and independent of spatial location. In our studies, we focus on the differential conductance spectra. According to the model of STS by Tersoff and Hamann [8], the differential tunneling conductance is proportional to the local electronic density of states of the sample and of the tip at energy $E=\mathrm{eV}$ measured from Fermi level. The effect of the voltage dependence of the transmission coefficient is often minimized by the use of normalized first derivative of the tunneling current versus sample bias, i.e. $(\mathrm{d} I / \mathrm{d} V) /(I / V)$.

The electronic structure of Ni clusters on Si substrate has also been studied using scanning tunneling spectroscopy. The STS spectra were acquired during topographic imaging by stopping the scan at selected points, sweeping the bias 
voltage through the regions of interest, and recording the $I-V$ characteristics or $\mathrm{d} I / \mathrm{d} V$ vs. $V$ (Fig. 4a, b, and Fig. 5a, b respectively). Some of these tunneling spectra (see Fig. $4 \mathrm{~b}$ ) show the opening of the semiconductor gap $\sim 1.0 \mathrm{eV}$ consistent with the silicon substrate.
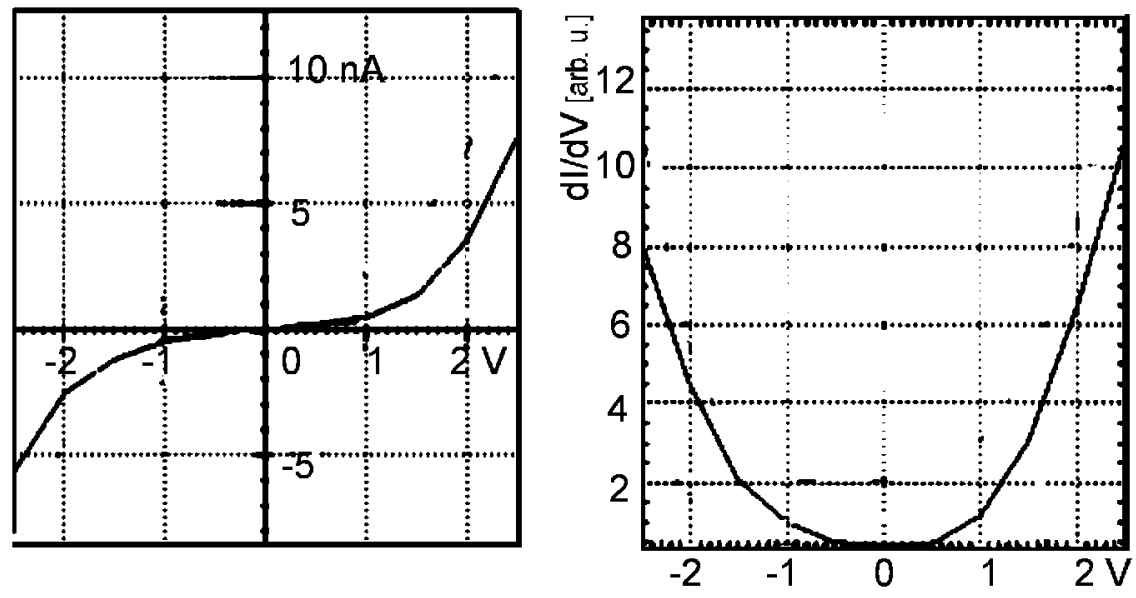

Fig. 4. $I-V$ (a) and differential conductance spectra (d $I / \mathrm{d} V)$ (b) for occupied and unoccupied electronic states obtained at positions taken away from the Ni particles.
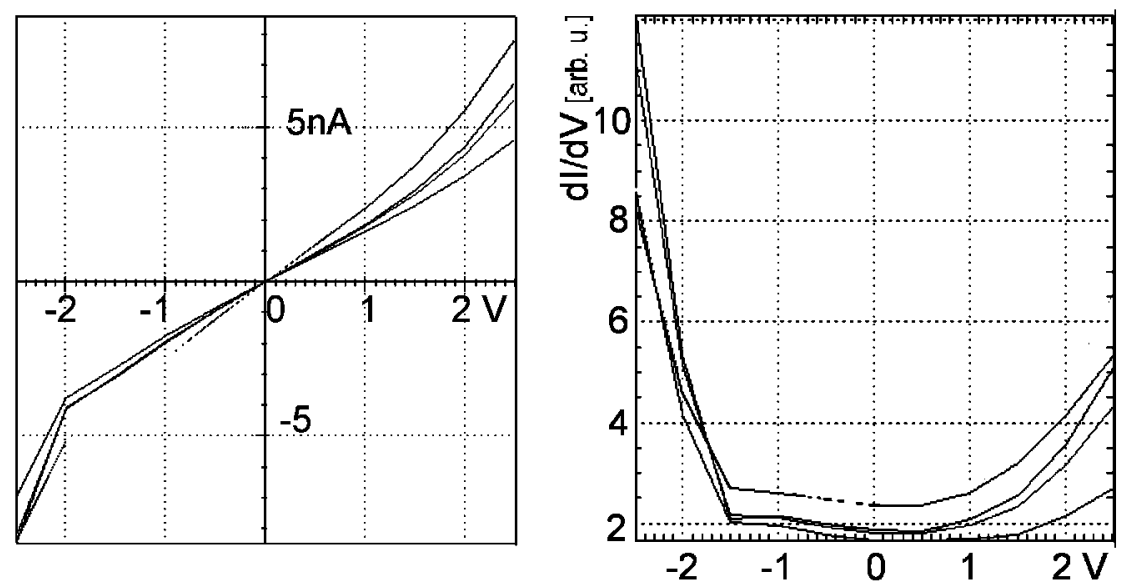

Fig. 5. $\quad I-V$ (a) and differential conductance spectra $(\mathrm{d} I / \mathrm{d} V)$ (b) for occupied and unoccupied electronic states obtained at five positions taken on the Ni particles.

For Ni clusters with diameter of $250 \mathrm{~nm}$ and a height of $6 \mathrm{~nm}$ we did not observe quantum size effect (QSE) at room temperature in the current-voltage characteristics on the top at the nickel clusters [9]. We believe that for small Ni clusters we will be able to observe QSE and electronic resonance peak near the 
Fermi energy $E_{\mathrm{F}}$ above the center of nanoparticles. Tunneling spectroscopy of the quantum structures exhibited features characteristic of the resonant energy level due to the finite size of the magnetic nanostructures.

\section{Conclusions}

Scanning probe methods were applied to study structural and electronic properties of Ni nanostructures prepared by nanosphere lithography. It was shown that nanosphere lithography can be a suitable preparation method of large-scale, periodic particle arrays. A great periodicity control allows the application of such nanostructures for catalytic growth of carbon nanotubes or magnetic storage media.

Experiments discussed here demonstrate the power of the STM/STS in probing the local density of states at an energy measured from Fermi level and surface morphology of periodic nickel nanoparticles. The work is under way to measure tunneling spectra at low temperatures down to $4.2 \mathrm{~K}$ with magnetic probe tips.

\section{Acknowledgments}

This work was supported by Poznań University of Technology Research Project TB 62-176/03-DS and by the State Committee for Scientific Research under grant No. 5 PO3B 10021.

\section{References}

[1] R.S. Becker, J.A. Golovchenko, D.R. Hamann, B.S. Schwartzentruber, Phys. Rev. Lett. 55, 2032 (1985).

[2] J.A. Stroscio, R.M. Feenstra, A.P. Fein, Phys. Rev. Lett. 57, 2579 (1986).

[3] R.M. Feenstra, J.A. Stroscio, J. Tersoff, A.P. Fein, Phys. Rev. Lett. 58, 1992 (1987).

[4] J. Rybczyński, U. Ebels, M. Giersig, Colloids and Surfaces A, Physicochem. Eng. Aspects 219, 1-6 (2003)

[5] R.M. Feenstra, J.A. Stroscio, A.P. Fein, Surf. Sci. 295, 181 (1987).

[6] R.J. Hamers, STM on Semiconductors, in series Scanning Tunnelling Microscopy I, Eds. H.-J. Guntherodt, R. Wiesendanger, Springer Ser. Surf. Sci., Vol. 20, Berlin 1992, p. 83.

[7] R.M. Feenstra, Phys. Rev. B 50, 4561 (1994).

[8] J. Tersoff, D.R. Hamann, Phys. Rev B 31, 805 (1985).

[9] B. Susła, M. Giersig, R. Czajka, M. Kamiński, Physica C 387, 221 (2003). 